$$
\text { CONF-900877-8 }
$$

To be published in Microbeam Analysis-1990

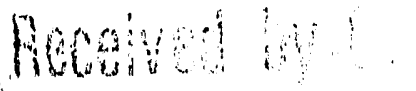

$\mathrm{BNL}--44490$

OCT 181990

DE91 000901

\title{
Biomedical Elemental Analysis and Imaging Using \\ Synchrotron X-Ray Microscopy
}

\author{
K. W. Jones, B. M. Gordon, G. Schidlovsky, P. Spanne, Xue Dejun \\ Atomic and Applied Physics Group \\ Brookhaven National Laboratory \\ Upton, New York 11973
}

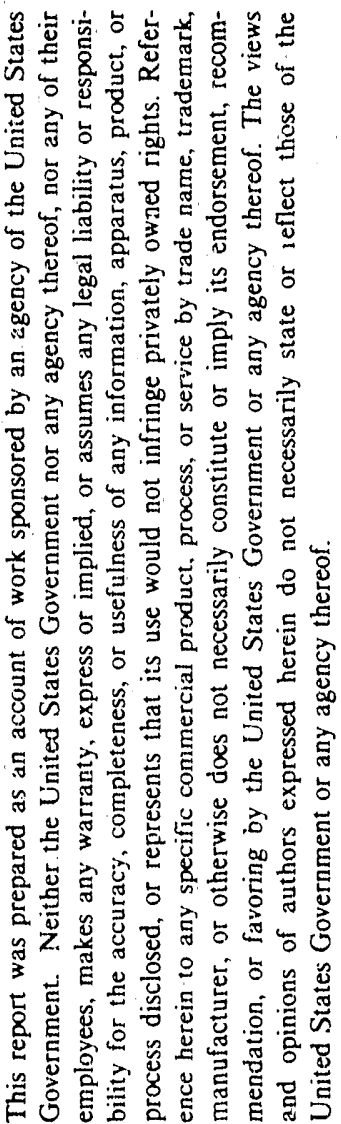

\author{
R. S. Bockman \\ The Hospital for Special Surgery \\ 535 East 70th Street, New York, New York 10021
}

\author{
A. J. Saubermann \\ Department of Anesthesiology, School of Medicine \\ Health Sciences Center, State University of New York \\ Stony Brook, New York 11794
}

\author{
Presented at \\ XIIth International Congress for Electron Microscopy \\ Seattle, Washington
}

August 13-17, 1990

By acceptance of this article, the publisher and/or recipient acknowledges the US Government's right to retain a nonexclusive, royalty-free license in and to any copyright covering this paper. 


\section{BIOMEDICAL ELEMENTAL ANALYSIS AND IMAGING USING SYNCHROTRON X-RAY MICROSCOPY ${ }^{1}$}

K. W. Jones, ${ }^{2}$ B. M. Gordon, ${ }^{2}$ George Schidlovsky, ${ }^{2}$ Per Spanne, ${ }^{2}$ Xue Dejun, ${ }^{2}$ R. S. Bockman, ${ }^{3}$ and A. J. Saubermann ${ }^{4}$

The application of synchrotron $\mathrm{x}$-ray microscopy to biomedical research is currently in progress at the Brookhaven National Synchrotron Light Source (NSLS). The current status of the $\mathrm{x}$-ray microscope (XRM) is reviewed from a technical standpoint. Some of the items considered are photon flux, spatial resolution, quantitation, minimum detection limits, and beam-induced specimen damage. Images can be produced by measurement of fluorescent $x$ rays or of the attenuation of the incident beam by the specimen. Maps of the elemental distributions or linear attenuation coefficients can be made by scanning the specimen past the beamı. Computed microtomography (CMT) can be used for non-destructive images through the specimen in either the emission or absorption mode. Examples of measurements made with the XRM are given.

The Brookhaven XRM uses continuous-energy bending-magnet radiation produced at the NSLS as the $\mathrm{x}$-ray source. The electron ring operates at an energy of $2.5 \mathrm{GeV}$ and a stored electron current of about $225 \mathrm{~mA}$ at the beginning of a fill and a current of about $100 \mathrm{~mA}$ at the end of the fill. The lifetime of a fill is approximately 24 hours, but there can be large fluctuations in this value depending on ring conditions. The $\mathrm{x}$-ray beam flux at the XRM specimen position is shown in Fig. 1 at a ring current of $100 \mathrm{~mA}$.

\footnotetext{
${ }^{1}$ Work supported by National Institutes of Health Biotechnology Research Resource Grant No. P41RR01838 and US Department of Energy, Office of Basic Energy Sciences, Division of Chemical Sciences, Processes and Techniques Branch, Contract No. DE-AC02-76CH00016.

${ }^{2}$ Atomic and Applied Physics Group, Brookhaven National Laboratory, Upton, NY 11973.

${ }^{3}$ The Hospital for Special Surgery, 535 East 70th Street, New York, NY 10021.

${ }^{4}$ Department of Anesthesiology, School of Medicine, Health Sciences Center, State University of New Yoik, Stoniy Brook, NY 11794.
} 
Emphasis is placed on retaining flexibility in the arrangement of the components in designing the XRM beam line. A monochromator prior to a focussing mirror can be used, if desired, to produce an image of the x-ray source with a demagnification of the source size by about a factor of two. The final beam size at the specimen is controlled by a set of $x-y$ adjustable slits and/or a pinhole. A set of four stepper-inotor driven tantalum slits can be used to produce collimated beams from a size of about $40 \mathrm{~mm}$ in the horizontal and about $10 \mathrm{~mm}$ in the vertical (consistent with the vertical opening angle of the source) down to a size of about $40 \mu \mathrm{m}$ set by the geometry of the source, pinhole, and specimen. A fixed geometry pin-hole collimator placed 3.5 centimeters from the specimen is used to define $x$ ray beams down to $1 \mu \mathrm{m}$ in size. Collimators of different sizes are used to cover the spatial range below $40 \mu \mathrm{m}$. It is sometimes desirable to replace the final collimator with a channelcut silicon monochromator. It can be used for simple experiments to produce monoenergetic beams with energies below the absorption edge for a major element or for chemical speciation using $\mathrm{x}$-ray absorption near-edge spectroscopy (XANES).

The photon flux is maximized by locating the XRM as close to the $\mathrm{x}$-ray source as possible $(9 \mathrm{~m})$. Flux values of about $3 \times 10^{8}$ photons $/\left(\mathrm{s}-\mu \mathrm{m}^{2}\right)$ integrated over the energy distribution of the synchrotron $x$-ray source are obtained with a $100 \mathrm{~mA}$ current of electrons in the storage ring. The specimen is moved with respect to the photon beam by use of computer-controlled stepping motors. The fluorescent $\mathrm{x}$-rays are detected with a $30 \mathrm{~mm}^{2}$ $\mathrm{Si}(\mathrm{Li}) \mathrm{x}$-ray detector placed at 90 degrees to the incident beam to minimize Comptonscattering. (The high-degree of polarization of the synchrotron $x$-ray source makes this possible.)

Some work has also been done using a Kirkpatrick-Baez microscope produced by Giauque et al. ${ }^{1}$ This instrument uses multilayer coatings to obtain high-throughput monoenergetic beams with energies from 6-14 keV. Spatial resolutions and photon fluxes obtained are very similar to the values mentioned above for collimated continuous radiation. 
To date, the use of the collimated XRM has the advantage of simplicity and broad range detection capability as compared to the focussed probe.

The sensitivity of the XRM for detection of characteristic $\mathrm{x}$ rays is found by use of specimens of known composition. For example, National Institute of Standards and Technology (NIST) Standard Reference Materials (SRM) 3171 and 3172 are multielement standards in liquid form. After deposition on filter paper, ${ }^{2}$ the fluorescent $\mathrm{x}$-ray spectra are analyzed to correlate the known elemental concentrations in units of $\mathrm{mM} / \mathrm{kg}$ with the number of characteristic $\mathrm{x}$ rays for given conditions of ring current, absorber thicknesses, detector solid angle, and efficiency. The measured values can be compared to calculations based on the theoretical values for photon flux produced by the synchrotron radiation method and known geometrical factors of the XRM. The results of sensitivity determinations made using the two methods are shown in Fig. 2. The calculated and experimental values are normalized at the maximum of the $\mathrm{K} x$-ray curve. The calculated values are about a factor of four higher in absolute value. This agreement is reasonable when the uncertainties in collimator area and alignment, as well as photon flux and energy distribution, are considered.

The specimen areal density is measured by comparing the scattered radiation intensity from the specimen to that from a standard of known thickness and similar composition. For work with tissue sections, a calibration curve is established by measurement of the scattered radiation from a set of polyimide foils of known areal density and then deducing the areal density of the unknown from this curve. The concentration values for the elements in the unknown are then easily found in units of $\mathrm{g} / \mathrm{g}, \mathrm{mM} / \mathrm{kg}$, etc. The same procedures are followed for specimens of bone where substantial amounts of $\mathrm{Ca}$ and $\mathrm{P}$ are present, although other types of standards have to be used. Absorption of the $\mathrm{x}$ rays in thick samples is accounted for using a modified version of the NRLXRF program. ${ }^{3}$ The accuracy of the 
concentration assignments is estimated at $\pm 10-15 \%$ while repeated runs on a single point show variation of $< \pm 5 \%$.

Detection limits assigned for tissue sections are $<0.02 \mathrm{mM} / \mathrm{kg}$ for elements aiound iron. These limits increase for lighter elements such as calcium. The detection limits for Kand L-X-ray detection are given in Fig. 3. We have investigated the detection limit for calcium by measuring a response curve in thin PVP sections with concentrations down to $12.5 \mathrm{mM} / \mathrm{kg}$. From the spectra obtainec, a value for the calcium detection limit of less than $0.1 \mathrm{mM} / \mathrm{kg}$ is obtained for a $300 \mathrm{~s}$ exposure. The present detection limits show that the XRM substantially improves on the limits attainable by electron-probe microanalysis. Further, several changes can be made in the existing apparatus which will improve detection conditions and reduce the detection limit.

The XRM is equipped $w^{i}$ h instrumentation and computer programs making computed tomography with spa tal resolution in the micrometer range possible. $6,7,8,9$ Measurements made using CMT are based either on fluorescent $\mathrm{x}$ rays or on differences in sample x-ray linear attenuation coefficients. Two types of CMT scanners have been developed, one is a high resolution first generation scanner utilizing a pericil beam ${ }^{6}$ and a single radiation detector, the other is a third generation scanner using a wide radiation field defined by a narrow slit and a photodiode array and a scintillator for the detection of $\mathrm{x}$ rays. The third generation scanner at present allows us to produce images with a spatial resolution of the order of $100 \mu \mathrm{m}$, but has potential for resolutions down to about $25 \mu \mathrm{m}$. The first generation scanner is used for imaging with much higher spatial resolutions. To date we have been able to generate a tomogram with a spatial resolution of approximately $1 \mu \mathrm{m}$ by defining a $1 \mu \mathrm{m} x$-ray beam with a collimator of the same type as is used for the fluorescence measurements. It is possible to produce such a small field at the sample position because the collimator can be placed as close to the sample as $1 \mathrm{~mm}$ during tomography, and the source to sample distance is of the order of $9 \mathrm{~m}$. The CM'T system is therefore not source- 
size limited at present. The CMT system can be used both for absorption tomography mapping the distributions of the linear attenuation coefficient in unsectioned samples and for elemental mapping using fluorescent $\mathrm{x}$ rays excited by the first-generation pencil beam.

The use of synchrotron $x$ rays for CMT has several advantages, mostly related to the very high flux of photons available. The high flux makes possible the use of monochromators for $\mathrm{x}$ rays, which has several interesting advantages compared to the broad $x$-ray spectra from conventional rotating anode $x$-ray tubes. The energy choice is important in minimizing the imaging time $e^{8}$ and gives the possibility for elemental mapping using photoelectric absorption edges or multiple energy CMT.

The interaction of high-flux x-ray beams with the specimen can cause damage to the specimen. We have looked at changes in the specimen morphology and in the change of $\mathrm{x}$-ray yield as a function of total fluence. For thin nylon foils severe damage is seen for a 300 s run, and a measurable decrease in scattered intensity is also observed for other types of plastic films. ${ }^{4}$ The measurable effects on biological materials are not found to be serious at present fluence levels, but they need to be carefully evaluated for individual specimens.

The use of the XRM to study the distribution of elements in a thin section of rat tibia or to measure the density distribution in a complete rat femur illustrates how the XRM can be applied in biological experiments. In particular, gallium nitrate is a therapeutic agent used to treat hypercalcemia in cancer patients. The mechanisms by which gallium acts to inhibit calcium release from bone is not known. Hence, knowledge of the distribution of the gallium in the bone and the bone density is important.

Bockman et al., ${ }^{5}$ used the XRM to map the distribution of gallium in the tibia of rats and showed that it accumulated preferentially in areas of highest bone metabolism. The work has been extended to study rachitic rats treated with gallium and vitamin D. Determinations were made of the concentraticns of calcium, iron, zinc, and gallium in line scans across the tibial shaft. A typical result from such a scan is shown in Fig. 4 for a rat 
that has been treated with both vitamin $D$ and gallium. It can be seen that there is a strong spatial variation in the trace elements across the diaphysis. The systematics of the changes and the dependence of the trace element values on gallium concentration can be found from such data. Detailed interpretation of the results obtained in this animal study is now in progress:

In-vivo serial measurements of the bone density of the rat femur as a function of treatment would be very useful for study of the gallium treatment. In principle, this can be done by the use of CMT. In order to demonstrate the approach a tomogram was made of a normal whole rat femur specimen. Bone studies by CMT on living arimals are contemplated for the near future.

A channel-cut Si monochromator, using diffraction off the (220) plane was mounted in the NSLS X-26C microprobe beam line between a four-jaw slit assembly and a Ta collimator with an opening of approximately $20 \times 20 \mu \mathrm{m}^{2}$. By placing the monochromator before the collimator, $x$ rays diffracted from planes other than the (220) plane could be prevented from reaching the sample and the detector. The monochromator was rotated with a remotely-controlled stepper-motor-driven rotator. The diffraction angle was set to an energy of $20 \mathrm{keV}$. The energy was verified by using a Mo foil (K-absorption edge at 20.0 $\mathrm{keV}$ ) and rotating the collimator while the $\mathrm{x}$ rays transmitted through the foil were counted. A $1 \mathrm{~mm}$ thick $\mathrm{CaF}_{2}$ (Eu) scintillation detector operated in current mode was used for the detection of the $\mathrm{x}$ rays transmitted through the sample. The sample, a formalin-fixed fomur from a normal rat, was placed on a sample stage consisting of two orthogonal stepper-motordriven translators carrying a rotator (cf. Spanne and Rivers ${ }^{6}$ ). A $10 \times 20 \mu \mathrm{m}^{2}$ radiation field was produced by tilting the Ta collimator relative to the $\mathrm{x}$-ray beam's direction of travel. Both the horizontal and the vertical dimensions of the beam were determined by scanning a $25 \mu \mathrm{m}$ thick $\mathrm{Cu}$ foil through the beam while measuring the number of $\mathrm{x}$ rays reaching the detector. This gives the edge spread functions in two directions for the system. The data 
collected during a translate rotate motion scheme of the sample through the $x$-ray beam were reconstructed using a filtered back projection algorithm 10 . The signal measured with the beam passing only through air was used for normalization.

Fig. 5 shows a projection image of the whole femur with the plane of the tomogram marked. This image was generated for orientation purposes, Fig. 6 shows the computed tomogram at the position marked in Fig. 5. The transverse spatial resolution in this image is approximately $10 \mu \mathrm{m}$ while the imaged slice is $20 \mu \mathrm{m}$ thick. The image shows in detail the fine structure in the bone. In the original image, small remains of bone marrow can also be seen, but this is not visible in the reproduction shown in Fig. 6. By sorting the pixel values in the tomogram into a histogram, a frequency function for the linear attenuation coefficient in the pixels can be produced and used for a further comparison of bones from rats that have been subjected to different diseases and therapies. In wet samples multiple energy imaging will allow a more definite separation of soft and bone tissues.

The XRM instrument described abuve is now being used for elemental analysis and imaging of a number of biological systems. For example, the distribution of essential trace elements ( $\mathrm{Fe}, \mathrm{Cu}, \mathrm{Zn}$, etc.), toxic elements ( $\mathrm{Pb}, \mathrm{Cd}$, etc.), and therapeutic elements such as $\mathrm{Ga}$ is currently being studied in bone, teeth, hair, and soft tissues in the dry state at room temperature and pressure. Provisions to study live tissue culture cells are being made. A facility to keep specimens at liquid nitrogen temperatures for study of low-Z diffusible elernents is in the planning stage. 


\section{$\underline{\text { References }}$}

1. R. D. Giauque et al., "Measurement of femtogram quantities of trace elements using an x-ray microprobe," Anal. Chem. 60: 855, 1988.

2. B. M. Gordon, et al., "The application of synchrotron radiation to microprobe trace el ament analysis of biological samples," Nucl. Instrum. Methods B45: 527, 1990.

3. J. W. Criss, NRLXRF, A Fortran Program for X-ray Fluorescence Analysis: Users' Reference Manual and General Documentation, Cosnic Program \#DOD-00065, The University of Georgia Office of Computing and Information Services, Athens, GA 30602.

4. K. Themner, et al., "Mass loss during $x$-ray microanalysis," Nucl. Instrum. Methods B49: 52,1990 .

5. R. S. Bockman et al., "Distribution of trace levels of therapeutic gallium in bone as mapped by synchrotron x-ray microscopy," Proc. Natl. Acad. Sci. USA 87: 4149 (1990).

6. P. Spanne and M. L. Rivers, "Computerized microtomography using synchrotron radiation from the NSLS," Nucl. Instrum. Methods B24/25: 1063 (1987).

7. P. Spanne and M. L. Rivers, "Microscopy and elemental analysis in tissue samples using computed microtomography with synchrotron x-rays," BioSci. Abs. 1: 101 (1988).

8. P. Spanne, "Microanalysis using computed microtomography and synchrotron $x$-rays," Presented at BioScience 90, Malmö, Sweden, April, 1990, BioSci. Abs. 3.

9. K. W. Jones et al., "Biomedical applications of synchrotron x-ray microscopy," in R. Moro and R. Cesareo, Eds., Proc. 2nd International Workshop on XRF and PIXE Applications in Life Scienre, Capri, June 1989, Singapore: World Scientific Publishing Co., 1990, 163.

10. G. T. Herman, in Image Reconstruction from Projections. The Fundamentals of Computerized Tomography, Academic Press, New York, 1980. 
FIG. 1.--The flux of $x$ rays produced by the NSLS bending-magnet source is shown as a function of $x$-ray energy. The units are the number of $x$-rays produced by a stored current of $100 \mathrm{~mA}$ which fall on a $1 \mu \mathrm{m} \times 1 \mu \mathrm{m}$ area at a distance $9 \mathrm{~m}$ from the $\mathrm{x}$-ray source. The values are given for a constant bandwidth of $1 \mathrm{eV}$. The effects of the beryllium windows used to separate the XRM from the vacuum conditions of the ring are shown.

FIG. 2.--Minimum detection limits for K- and L-x-ray detection are given as a function of atomic number, Z. Values scaled from those reported by B. M. Gordon and K. W. Jones, Nucl. Iristrum. Methods B10/11: 293 (1985) are given to indicate the agreement with calculations for a focussing XRM. The proton curve shows the values attained using a proton microprobe and proton-induced $\mathbf{x}$-ray emission (PIXE).

FIG. 3.--The sensitivity for elemental concentrations using the XRM is shown as a function of atomic number. The beam size is $8 \mu \mathrm{m} \times 8 \mu \mathrm{m}$ and the ring current is $100 \mathrm{~mA}$. Experimental points were determined using thin standards. The curve was calculated from knowledge of the photon flux and experimental parameters. The detector solid angle was $1.2 \times 10^{-3} \mathrm{sr}$.

FIG. 4.- A line scan across a rat femur section taken from a rachitic rat treated with Vitamin $\mathrm{D}$ and gallium. The beam size was about $5 \mu \mathrm{m} \times 8 \mu \mathrm{m}$. In this case the concentration values are referenced to the average calcium value taken as $20 \%$ of the bone's wet mass. Values for $\mathrm{Ca}$ (triangles), $\mathrm{Fe}$ (dashes), $\mathrm{Zn}$ (dots), and $\mathrm{Ga}$ (solid line) are given.

FIG. 5.--X-ray projection image of a rat femur generated with $20 \mathrm{keV}$ synchrotron $\mathrm{x}$ rays. FIG. 6.--X-ray absorption tomngram of a rat femur imaged with $20 \mathrm{keV}$ synchrotron $\mathrm{x}$ rays. The matrix size is $285 \times 285$; the pixel size is $15 \mu \mathrm{m} \times 15 \mu \mathrm{m}$. 
FIG. 1
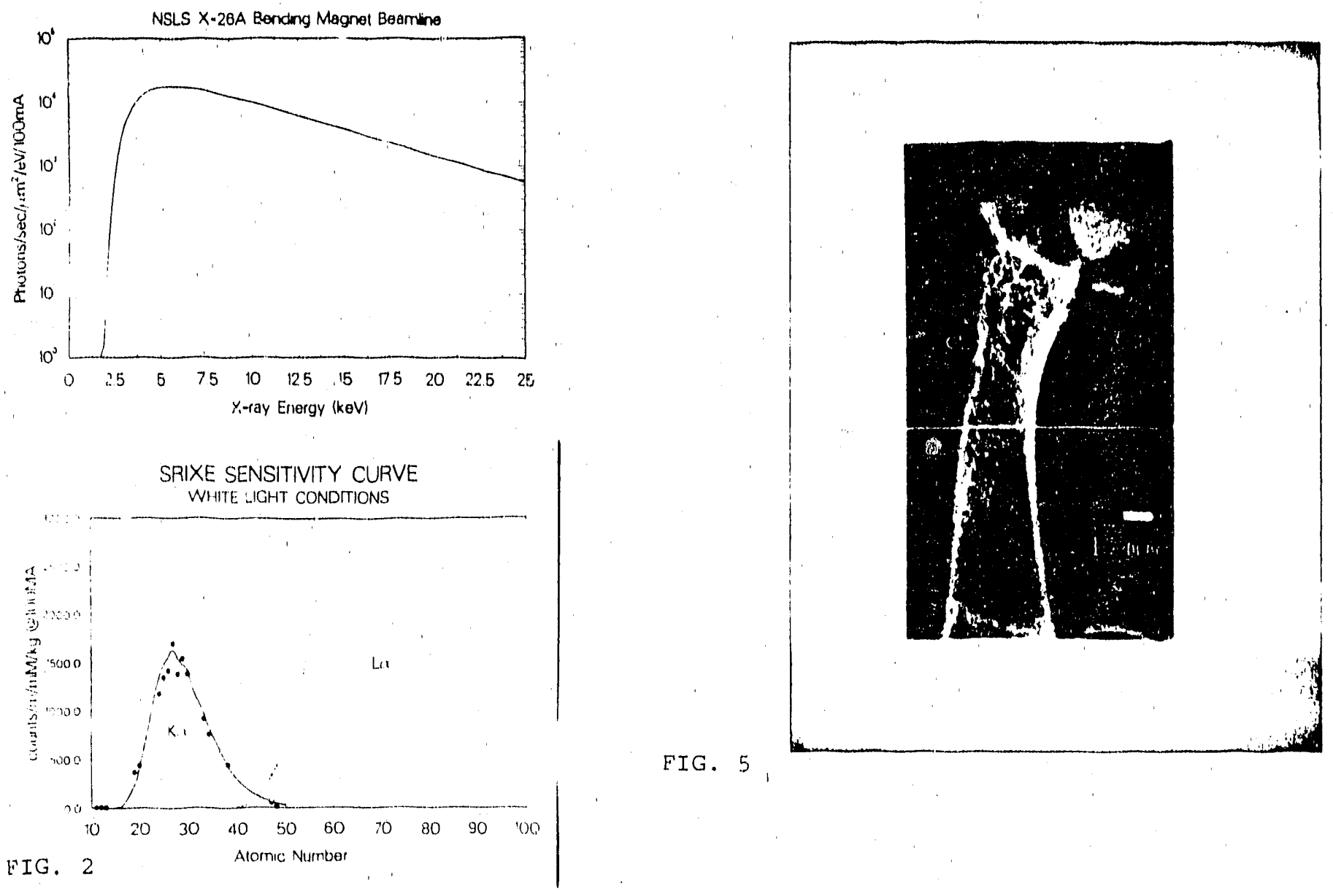

FIG. 5

SRIXE MINIMIUM DETECTION LIMIT WIIIE LGIHT CONDITIONS:

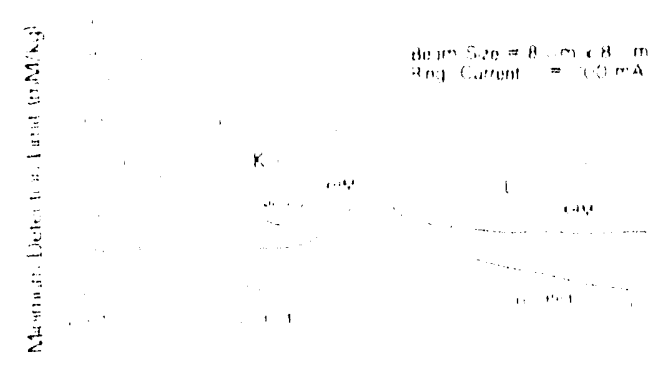

EIG. 3

Alorrit: 'Herour'

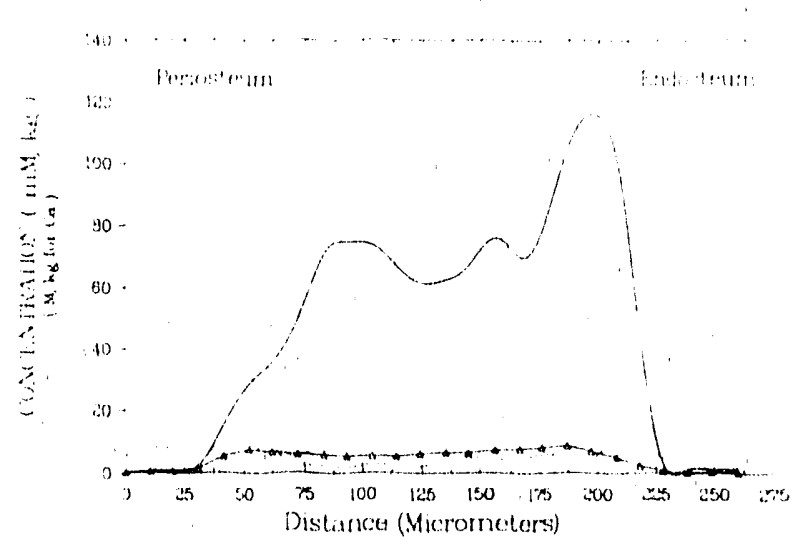

FIG. 6

EIG. 4 

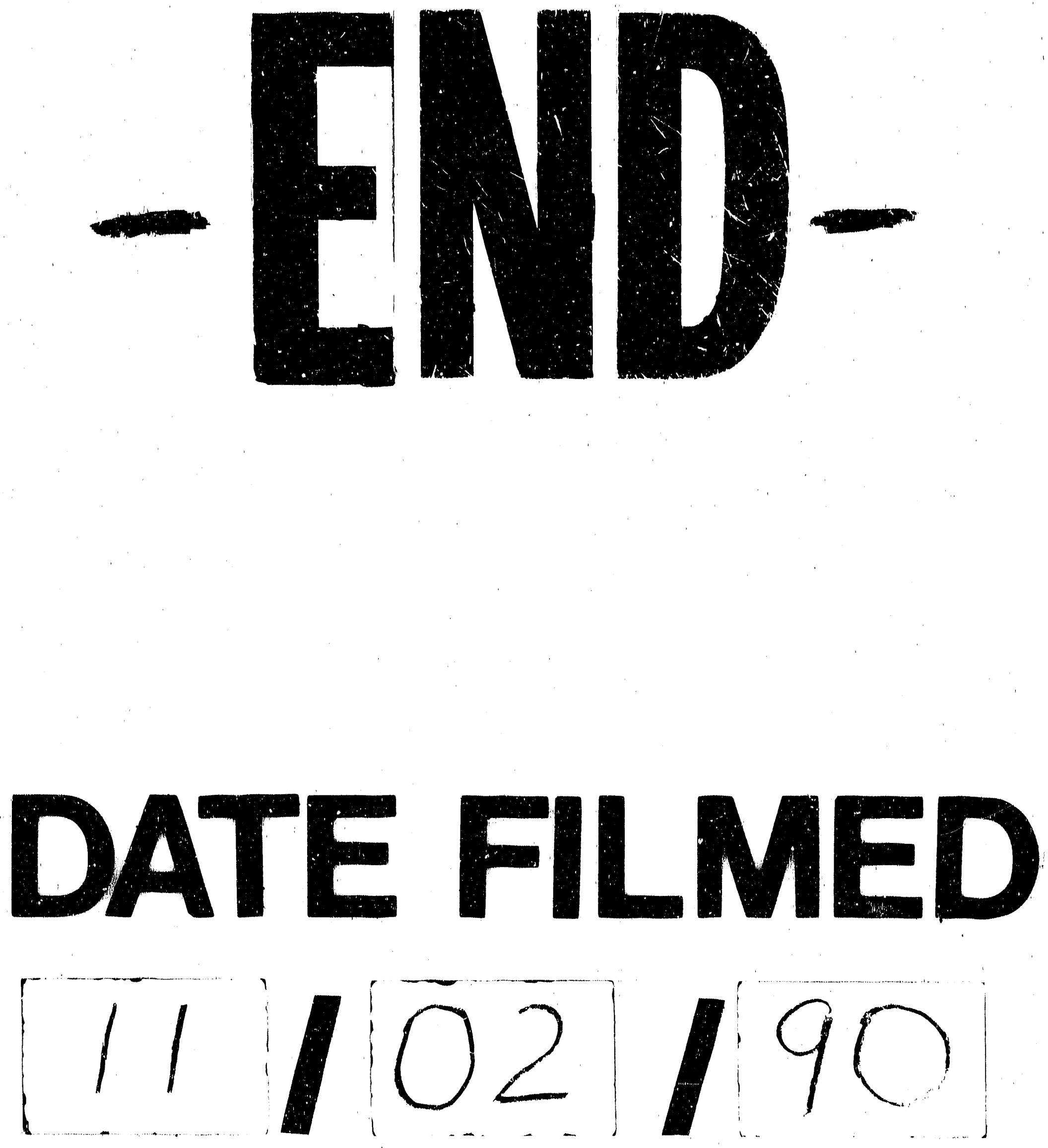

1 